\title{
TOWARDS A FRAMEWORK FOR CREATION OF DYNAMIC VIRTUAL ORGANIZATIONS
}

\author{
Luis M. Camarinha-Matos ${ }^{1}$, Ivan Silveri ${ }^{2}$, \\ Hamideh Afsarmanesh ${ }^{3}$, Ana Inês Oliveira ${ }^{1}$ \\ ${ }^{l}$ New University of Lisbon, PORTUGAL, cam@uninova.pt, aio@uninova.pt \\ ${ }^{2}$ TXT e-solutions SpA, ITALY, ivan.silveri@txt.it \\ ${ }^{3}$ University of Amsterdam, THE NETHERLANDS, hamideh@science.uva.nl
}

\begin{abstract}
Effectiveness of the virtual organization (VO) creation process is the base for the possibility of having truly dynamic VOs in response to collaboration opportunities. A realistic approach to materialize agility in VO creation is the assumption of a VO Breeding Environment. In this context, a discussion of the process and suggested functionalities towards a VO creation framework are presented.
\end{abstract}

\section{INTRODUCTION}

The possibility of rapidly finding a set of partners that best fit a business opportunity and quickly configure them into a collaborative network to exploit that opportunity seems indeed a desirable scenario to face the challenges of market turbulence. The same idea is also very appealing in other non-business oriented contexts. An extreme case being the incident management and disaster rescuing processes, when it is necessary to very rapidly engage and coordinate activities of a large number of entities (e.g. fire brigades, police, hospitals, local government, non-governmental organizations). This very idea of groups of organizations being able to rapidly configure themselves into some form of mission/goal-oriented collaborative form embeds the notion of great agility.

Finding the right partners and establishing necessary conditions for starting the collaboration process might however be costly and a time consuming activity, and therefore an inhibitor of the aimed agility. Among others, obstacles include lack of information (e.g. non-availability of catalogs with normalized profiles of organizations) and lack of preparedness of organizations to join the collaborative process. Overcoming the mismatches resulted from the heterogeneity of potential partners (e.g. different as in infrastructures, corporate culture, methods of work, and business practices) requires considerable investment, building trust, a pre-requisite for any effective collaboration, is not straight forward and requires time.

Furthermore, partners' selection is not a simple "optimization" problem. More than a matching process based on potential and abilities (e.g. competencies, capacities, recommendations), many other factors, some of them of subjective nature (e.g. personal preferences and established trust based on previous experience), suggest that fully automated processes are not at all a realistic approach. It is rather 
preferable to conceive a computer-assisted framework to help the human planner in making decisions.

The ECOLEAD project is designing a VO creation framework which represents an approach to minimize the mentioned difficulties by conceiving the VO creation within a VO Breeding Environment context. After a brief analysis of related work, the following sections summarize the approach being followed in ECOLEAD as well as the preliminary results of this ongoing initiative.

\section{PAST APPROACHES}

Three approaches are so far addressed in the $R \& D$ as alternatives for VO creation.

\subsection{Manual or assisted approach}

Manual or assisted approaches in VO creations are derived from the traditional methods adopted in working group creation for big organizations or for extended enterprises, mostly based on "competency" matching.

The adoption of a competency-based approach in Human Resource Management was initially determined by the acknowledged inability of traditional psychological and cultural tests in predicting successful job performances. In the early seventies, McClelland and his research group demonstrated that traditional measures, primarily based on candidates' cultural background, were not meaningfully correlated to excellence on job and, moreover, the results were strongly biased by racial, social and sexual prejudices [10]. McClelland's approach was based on two fundamental rules: (1) To compare groups of excellent or best performers with group of average performers in order to determine which characteristics could be associated to successful job performances. (2) To elicit operational cognitive schemata and behaviors that can be related to the job performance on a causal and objective basis.

The competence-based approach has received a wide consensus in Human Resources Management practices because it allows overcoming the typical pitfalls of the traditional job-based approach where the basic mechanism to identify the right person for the right job was the following: (1) To describe precisely the set of tasks, activities and responsibilities characterizing a given job; (2) To find the individual that best fits the job description: this is done applying the matching selection.

The set of required skills of a potential partner is usually called "profile". A skill is the ability to perform a physical or mental task, i.e. what we can do. A competence is knowledge and / or skill, plus capacity to apply it and the ability to make others applying it. It is characterized by managerial capabilities and personal qualities. Competency is strictly connected to work situations (e.g. information diffusion in inter-functional team). It is how we behave when performing a task, how we go about it and the application of our knowledge and skills.

A profile is moreover influenced by other external factors, not directly related to the candidate's skills, but which are affecting its matching index: namely, the behavioral aspects. These are very relevant and cannot be classified and categorized in the form of competence, but they should be traced in historical records that must be considered when a candidate's ranking is made. Such aspects could be for instance:

- Past performance experiences: how was the candidate behavior, for instance if it was collaborating, active, proactive in providing contributions as well as in 
performing the task.

- Human Competencies: what affects the human being sphere, if the candidate is keen in team working, etc.

The candidate's matching/selection is typically done in two steps: (1) a matching algorithm is applied to the candidates' list based on the skills that can be well defined (the competencies), (2) these candidates are filtered applying the second type of competencies, which affect the human behavior.

Most of the considerations made in the context of human resources management apply as well to the selection of organizations as partners for a VO. Other aspects to consider include the interoperability readiness, size of organization, "robustness" of its skills base (i.e. critical mass), geographical location, costs, etc.

A typical difficulty is the access to reliable profile and performance information as well as the time spent with the preliminary filtering of potential candidates. An earlier example of attempt to move from a manual approach to a computer assisted one can be found in the PRODNET project [6]. Recently there has been a considerable effort put in the so-called electronic procurement [4]. The main objectives in this area include the definition of "normalized" procedures for public announcement of business offers, reception, and management of bids. Standardization is in fact the main obstacle in electronic procurement. The VO partners' search and selection activity shares several similarities with the classic electronic procurement. Both areas require the identification of potential suppliers / partners to be addressed, the adoption of normalized specification of requirements and bids, management of directories of potential partners, management of bids, and decision support functionalities.

\subsection{Multi-agent based approaches}

A growing number of works are being published on the application of multi-agent systems (MAS) and market-oriented negotiation mechanisms for the Virtual Enterprise (VE) formation [4].

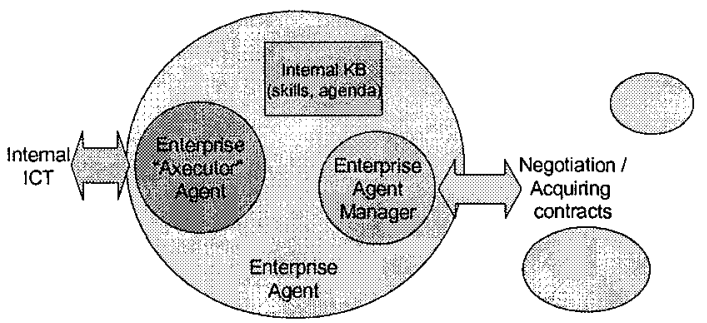

Figure 1 - Enterprise represented as an agent

One early example can be found in [13]. This work assumes a virtual market place where enterprises, represented by agents that are geographically distributed and possibly not known in advance, can meet each other and cooperate in order to achieve a common business goal. A MAS architecture is proposed to model the electronic market to support the formation of the VO. In addition to the agents representing the enterprises, there is a market agent - coordinator or broker - that is created and inserted in the MAS community when a business opportunity is found. A multi-round contract-net protocol is followed and the most favorable bids are selected based on a multi-criteria mechanism and constraint-based negotiation. Examples of considered criteria are lower-cost, higher quality, higher availability, etc. Utility values are associated to each of these criteria and a linear combination of attribute values weighted by their utility values is used. Multiple negotiation rounds can take place. At the end of each round bidder agents 
receive indication whether their bids are wining or loosing and a rough qualitative justification, allowing them to change the parameters of their proposals.

A similar work is found in [9] where a more detailed analysis of the problem of goal decomposition, leading to a hierarchy of VE goals, is done. In addition to the enterprise agents and VE coordinator agent (broker), an information server agent is introduced to keep public information related to common organizational and operational rules, market environment, enterprises and products / services provided, etc. The need for a common ontology to support the communication among agents is explicitly introduced and a multi-attribute, constraint-based negotiation / selection process is implemented. The work described in [14] identifies the need for yellow pages agents that are responsible to accept messages for registering services (similar to the information agent server mentioned above). They also consider the concept of Local Area, a quasi-physical division of the network that can be controlled by a local area coordinator. This is a similar concept to the Local Spreading center first introduced by the HOLOS system [11, 12]. Finally [8] elaborates further on the application of market-oriented principles, with particular reference to the principles of general equilibrium in micro-economics.

More recent works have attempted to progress with new negotiation protocols, auction mechanisms, distributed matching processes, etc. In order to improve the effectiveness of the contracting process and to dynamically form VOs the need to develop forms of e-contracting has been identified. Several significant characteristics for the e-contracting process can be found in [1]. These proposals are however still limited by a number of factors which affect their practical implantation including: (i) Lack of common standards and ontologies, a situation difficult to overcome in a general "open universe" of enterprises; (ii) None of these proposals takes properly into account more subjective facets (soft computing issues) like trust, commitment, successful cooperation history, etc.; (iii) In general they pay little attention to the implantation aspects and the management of the yellow pages / market place; (iv) Security issues in the negotiation process are not addressed, which is a critical point as the agents are only partially cooperative (they might be selfinterested, competitive, and even exhibit antagonistic behavior). On the other hand, the attempt to reach a fully automated decision-making process, although an interesting academic exercise, is quite unrealistic in this application domain. Furthermore, as agents are designed and developed independently, it is quite difficult to guarantee their coordination unless common rules ("social laws") are adopted. Agent-based approaches can lead to an interesting simulation tool.

\subsection{Service federation based approach}

Figure 2 illustrates the basic principles behind the service federation approach [5]. According to this model, companies (potential members of the virtual organization) are considered as "service providers", i.e. the potential collaborative behavior of each company is "materialized" by a set of services. The approach assumes the existence of one entity that keeps a catalog of services where service provider companies publish their service offers. This entity is sometimes called a "service market", a "service promoter node", or even "service portal". Regardless the different implementation approaches the general three major functions - publish, discover, invoke - are usually considered.

This approach reflects an indirect partners' selection - what is selected is the 
service (not the provider), i.e. the immediate task is the composition (or orchestration) of complex services, not the consortia. Partners are implicitly selected via the specific services that are chosen. One example of this approach applied to the tourism sector can be found in [2].

Although the "popularity" of the web services paradigm give this approach some relevance, there are still a number of limitations in the current service model including: Are services always available? How is the workload balanced? What is the level of awareness of the service provider? Can all skills be represented as services? Does it make sense to consider specific services for the partner search / negotiation phase? Furthermore, most example developments so far are for ecommerce, not for collaborative activities.

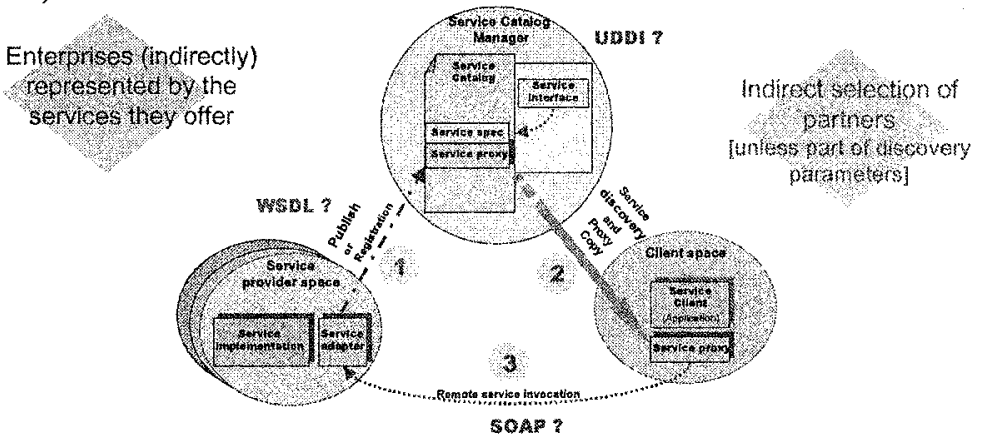

Figure 2 - Service federation approach

One important feature for the service catalog is the opportunity to provide the broker with an intelligent search / selection filtering lookup functionality based on the services attributes. Another main issue is the access rights, that is, who are allowed to access the information in the catalog. The service providers shall keep the autonomy and the right to specify whom and under which conditions has access rights to their registered services.

\section{VO CREATION PROCESS}

In ECOLEAD the VO creation process is considered to happen in the context of a VO Breeding Environment (VBE) [3, 5, 12]. This long term collaborative association is composed of organizations that are prepared to collaborate and thus rapidly respond to a collaboration opportunity.

As illustrated in Fig. 3, it shall be noted that VBE creation and VO creation are different processes, triggered by different motivations. A VBE is created as a long term "controlled border" association and its members are recruited from the "open universe" of organizations according to the criteria defined by the VBE creators or administrators. A VO is a temporary organization triggered by a specific business / collaboration opportunity. Its partners are primarily selected from the VBE members. In case there is a lack of skills or capacity inside the VBE, organizations can be recruited from outside. Considering that each company might have its list of "acquaintances" outside the VBE (i.e. other partners or former partners in activities outside the VBE), the search space can be seen as first composed of the VBE members, then with less probability by acquaintances of the VBE members (a kind 
of "extension" of the VBE borders), and finally by the "open universe" of organizations. For difficulties of preparedness, trust, etc, this last category will, of course, be the last resort.

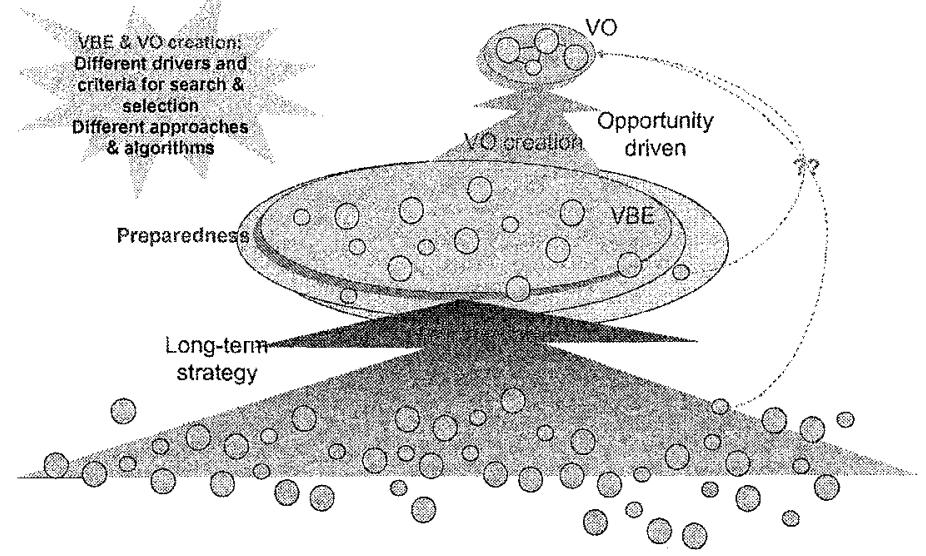

Figure 3 - VO creation in a VBE context

In this context the following main steps (Fig. 4a) are suggested for the VO creation process:
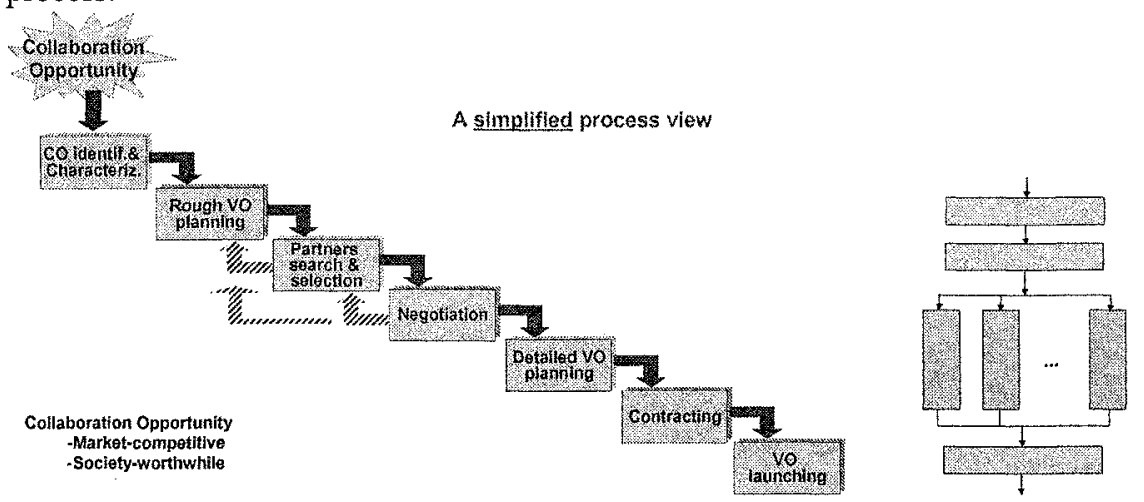

Figure 4-a) VO creation process

b) Alternative solutions

- Collaboration Opportunity Characterization: this step involves the identification and characterization of a new Collaboration Opportunity $(\mathrm{CO})$ that will trigger the formation of a new VO. A collaboration opportunity might be external, originated by a (potential) customer and detected by a VBE member acting as a broker. This is the case, in the business world, when the possibility to satisfy a customer, gaining money, rises from collaboration between members. Some opportunities might also be generated internally, as part of the development strategy of the VBE. This could happen in order to improve the VBE overall quality and competitiveness or the profitability of some members. As an example of this class of collaborations it is the case when a manufacturer company would like to reduce the failure rate of its production process. In order to do this it will approach a problem solving process involving other members of the $\mathrm{VBE}$, which have equal or similar production 
process.

Four main collaboration modalities were identified, namely: (1) Collaborative business process (BP) model, (2) Project model, (3) Problem solving model, (4) Adhoc collaboration model.

A collaborative $\boldsymbol{B P}$ model can be defined as a set of heterogeneous activities normally distributed in cross-organizational sub-processes. These activities can be categorized as in the following: Automatic Activities, implemented by ICT services; Interactive Activities, implemented by Collaborative Work plus ICT services; Cooperative Activities implemented by Co-Work; Manual Activities, implemented by Humans; Decisional Activities, followed by "or" branches, alternatives (Fig. 5).

A collaborative project model can be defined as the support for multi-projects towards the definition of a work break down structure (WBS), composed by multiprojects, workpackages, tasks and activities and the support for the human resource management where Human Resources belongs to multi-organizations (Fig. 6). VOs, which will adopt this model, will need a set of supporting tools: Technical/Financial Control, control primitives for progress, technical (percentage of completion) and financial (budget); Co-Work Integration, integration with co-work services, collaboration measurement and rewarding; Knowledge Management Integration, integration with document and multi-media material exchange/sharing; project management facilities, nice presentations, re-planning and re-scheduling facilities for project managers.

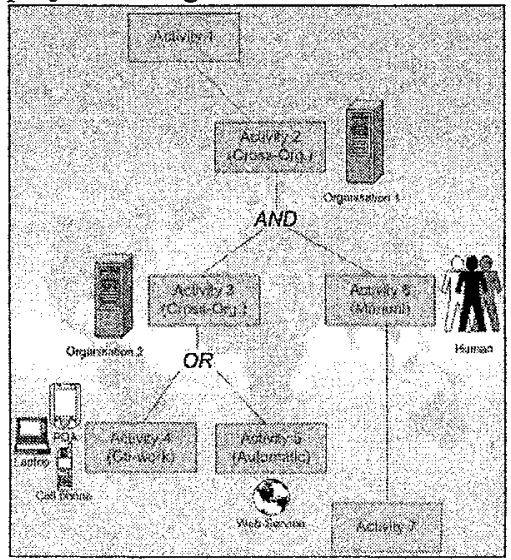

Figure 5 - Collaborative BP

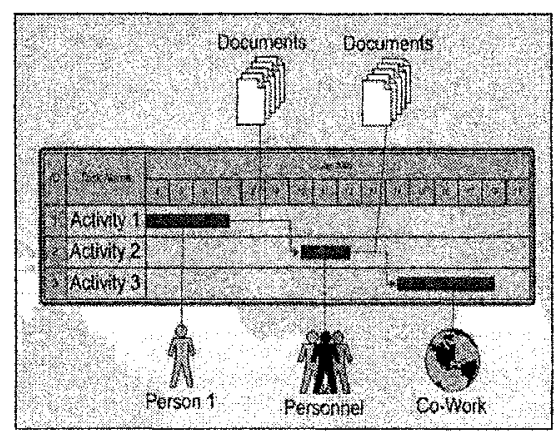

Figure 6 - Collaborative Project

In a collaborative problem solving a roadmap model must be established defining the as-is situation and the to-be scenario, performing the gap analysis and identifying the working groups (Fig. 7). In this case a VO will need: a Mediated Collaboration Model, where the mediator identifies contributors, collects and evaluates contributions; Collaboration Measurement \& Reward, that is identification of value metrics, rewarding of experts contributing decisively towards the roadmap's goal achievement; Personal Time/Work Organizer, that is the possibility to integrate Project-Problem Solving Ad-Hoc collaboration sessions and/or off-line e-work; Specific Tools for Problem Solving, that is the integration with specific Problem Solving tools, like Diagnosis Tools, Case-based Reasoning, Expert Systems and Continuous Improvement. 
The ad-hoc collaboration model can be very useful when big organizations, not used to tightly collaborate one with the other, are required to joint their efforts in order to rapidly give a quick response to an external request (e.g. like fire brigade and red cross in an emergency case) (Fig. 8); in this model only few persons are required to take decisions and in this way addressing their organizations towards the common goal. Supporting to this model is given by: Integration and Composition of Co-Work, several Co-Work services to be combined and integrated as atomic services; Co-Work Logging, primitives for multi-media Co-Work logs recording (text, speech, image, video); Co-Work Analysis, advanced services for Co-Work logs analysis (speech-to-text; MPEG analysis); Ubiquitous Access, as far as possible to integrate the Co-Work services with PTO/PWO (personal time organizer/ personal work organizer) in an AAA (Anybody-Anywhere-Anytime) scenario.

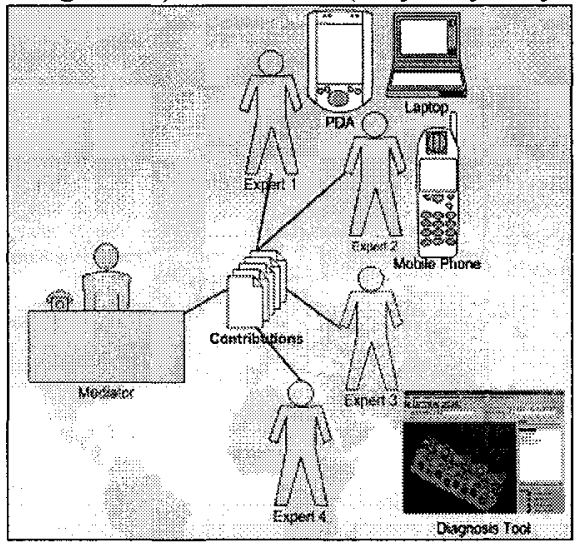

Figure 7 - Collaborative Problem Solving

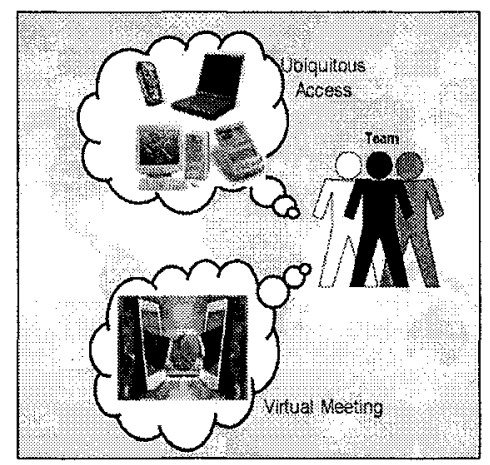

Figure 8 - Ad-hoc collaboration

Therefore the main issues to consider in this initial step are: Identification and categorization of the $\mathrm{CO}$ (external/explicit, internal/implicit), Collaboration modality, $\mathrm{CO}$ representation, $\mathrm{CO}$ feasibility analysis, Interaction with customer, Support (rapid) quotation / bidding, etc. The main actors involved in this step are the broker and the customer.

- Rough VO planning: determination of a rough structure of the potential VO, identifying the required competencies and capacities, as well as the organizational form of the $\mathrm{VO}$ and corresponding roles.

At this stage it is important to define the partnership form which is typically regulated by contracts and cooperation agreements. Fig. 9 illustrates some typical organizational forms for collaborative consortia [4].

Case 1: Explicit consortium. Collaboration is regulated by a joint contract with the customer and a consortium agreement. The Client cares about who is part of the consortium.

Case 2: Internal consortium. There is a contract between one representative of the consortium and the Client. The Client doesn't necessarily know about the way the consortium is organized. The consortium is also formalized using an agreement and an internal contract. Only one partner (the one that signs the contract) is committed to the Client. The other partners are committed to the one that signs the contract.

Case 3: Sub-contracting. There is a contract between one partner and a client and 
subcontracts between this partner and the other service / product providers. The Client doesn't necessarily know about the way the contracted partner is organized.
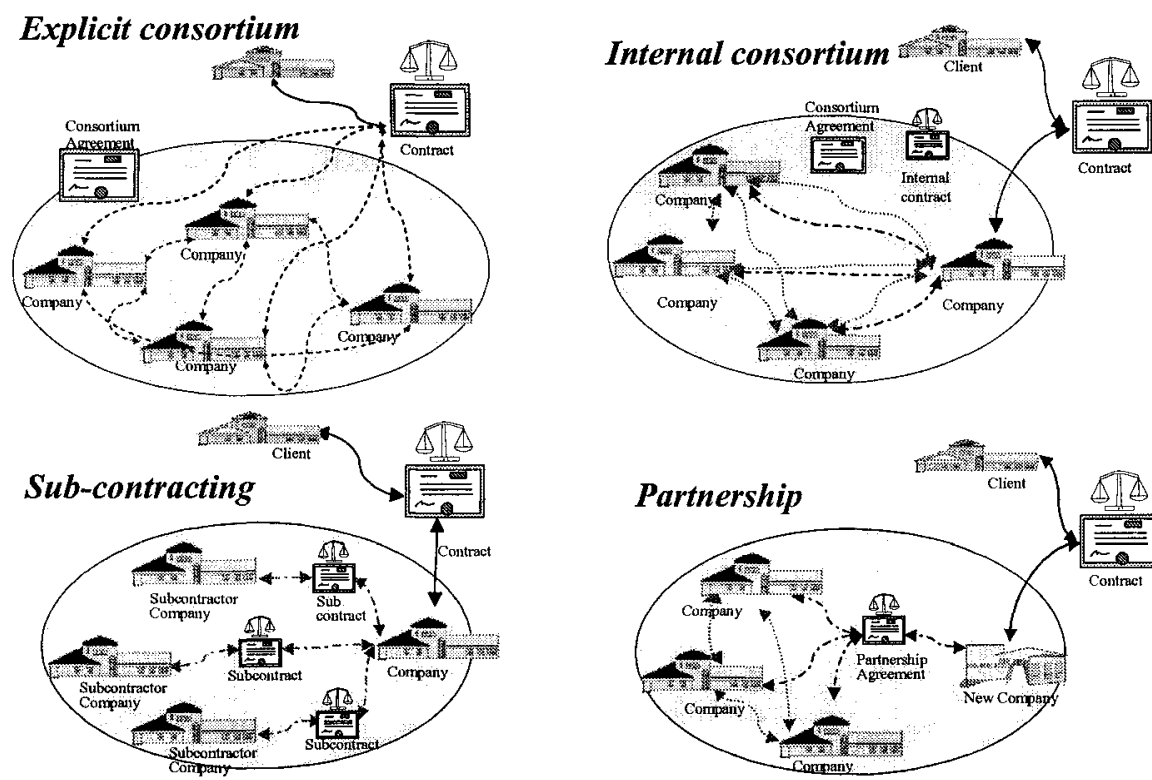

Figure 9 - Different types of collaborative consortia

Case 4: Partnership. The partnership creates an entity (new company) using a partnership agreement. The new company establishes a contract with the Client. Only the new company is committed to the Client. The partnership may continue after the end of the initial client contract.

Cases 1 and 2 are the most typical situations for dynamic VOs.

Other issues to be considered at this stage: Identification of needed competencies and capacities, Rough VO structure / topology / levels and associated roles, macro governance rules, Representation of rough VO model, Top-down (planning) vs. bottom-up (emerging), Simulation (to assess different configurations), etc. Main roles involved in this step: Broker role and VO planner role.

- Partners search and selection: perhaps one of the most addressed topics in past research, this step is devoted to the identification of potential partners, and their assessment and selection.

Issues to consider: Elements for search and selection (technical, economical, reliability indicators, preferences); matching algorithms; (multi-criteria) selection criteria; optimization; assessment (preparedness, etc), consideration of collaboration history / record; external search (if the internal offer is insufficient); etc.

Multiple strategies / algorithms can be considered to support this task (Fig. 4.b). For instance a frequent solution follows a top-down approach in which the VO planner designs the VO, deciding on which roles and selecting the partners that best fit his plan. An alternative would be a bottom-up (competition) approach in which the VO planner or the broker announces the collaboration opportunity to the VBE and waits till some consortia spontaneously form (by the initiative of some members) and then analyzes their global bids. 
Nevertheless, it is not only in the creation phase that the selection is important, as in the operation phase it also might be needed some new partner to execute some sort of task that no other partner can perform; or even there might be the case where a partner needs to be replaced. The main roles involved are the VO planner role, the VBE member role, and in some cases the broker.

- Negotiation: is an iterative process to reach agreements and align needs with offers. It can be seen as complementary to the partners' selection process and might in fact require going back to the previous step(s) if a solution cannot be found with the current configuration of partners.

Important issues to consider at this stage include: Determination of the objects of negotiation; Negotiation protocols; Decision making process and corresponding parameters; Representation of agreements; etc. BP negotiation, i.e. BP refinement and assignment to partners may also be considered at this stage (if not in the detailed VO planning). The main roles intervening in this step are the VO planner role, and the VBE member role.

- Detailed VO planning: once partners have been selected and collaboration agreements are reached, this step addresses the refinement of the VO plan and its governance principles.

This step involves the business / collaboration process modeling (depending on the type of collaboration - BP, collaborative project, collaborative problem solving, ...); Final VO representation; Assignment of roles and responsibilities; Definition of sharing principles, access levels (assets/resources, IP, benefits,...), preliminary operating policies; etc. The main contributors to this step are the VO planner role, and the VBE member role.

- Contracting: involves the formulation and modeling of contracts and agreements as well as the contracting process itself, before the VO can effectively be launched.

A contract is an agreement between two or more competent parties in which an offer is made and accepted, and each party benefits. A contract defines the duties, rights and obligations of the parties, remedy clauses as well as other clauses that are important to characterize the goal of the contract. An agreement is an arrangement between parties regarding a method of action. The goal of this arrangement is to regulate the cooperation actions among partners, and it is always associated to a contract.

In addition to the definition of the types of contracts and their representation, this step needs to deal with: Contracting process (manual, e-contracting); Contract enforcement mechanisms and institutions; Legal issues; etc. This step needs to be performed in close interaction (in parallel) with the Negotiation and Detailed VO Planning steps. Main roles involved: VO planner role, VBE member role, and VO coordinator.

-VO launching: the last phase of the VO creation process, i.e. putting the VO into operation, is responsible for tasks such as configuration of the ICT infrastructure, instantiation and orchestration of the collaboration spaces, assignment and set up of resources / activation of services, notification of the involved members, and manifestation of the new VO in the VBE. Main roles to be involved in this step: VO coordinator, VBE member role, and possibly the VBE administrator. 


\section{VO CREATION FUNCTIONALITIES}

Based on the process and requirements described above, a number of support functionalities and information models can be identified, as illustrated in Fig. 10.

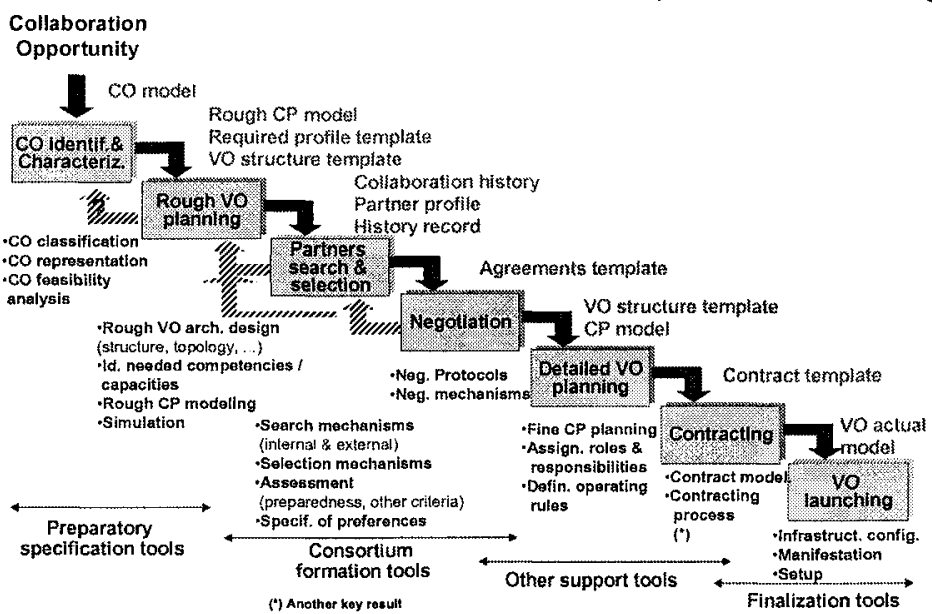

Figure 10-Main functionalities and support models for VO creation

As preparatory specification tools and mechanisms (supporting the first two steps), the following examples have been identified: $\mathrm{CO}$ characterization and representation tool; CO feasibility analysis; Rough VO architecture design (structure, topology, main roles); Identification of needed competencies and capacities; Rough collaboration process modeling; and Simulation for preliminary assessment. Main information models for this phase include: Collaboration opportunity model, Rough collaboration process model, required partner(s) profile template, and VO structure template.

As consortium formation tools (next two steps), we can consider functionalities and mechanisms for: Partners search and selection (internal to VBE and external); Assessment of partners preparedness / fitness; Specification of preferences; Negotiation protocols and methods. Main information models for this phase include: A record of collaboration history, members profiles, agreements template, etc.

Some other support tools are important to help the detailed planning and contracting, such as for instance: Mechanisms to assign roles and responsibilities; Definition of operating rules for the VO; Contract modeling; and support the contracting process. Main information models for this phase include: VO structure template, Collaborative process (or business process) model, Contract template.

Finally as finalization tools we could refer: Configuration and setup of the infrastructure and resources; Manifestation of the VO; Notification of the partners; etc. Main information model for this phase is the detailed or actual VO model.

\section{CONCLUSIONS}

The time and amount of resources consumed during the VO creation process whenever a business/collaboration opportunity is acquired, give a good indication of 
the level of agility of a collaborative ecosystem. The effectiveness of this process mainly depends on the availability of adequate information about potential partners and their level of preparedness for VO involvement. The existence of a VO breeding environment facilitates these requirements and thus enables truly dynamic virtual organizations. The ECOLEAD approach to VO creation is developed under such assumption and proposes a detailed process covering all required steps from the identification of the collaboration opportunity till the actual launching of the VO that will exploit that opportunity. Ongoing research is aimed at developing a full VO creation framework and corresponding support tools.

Acknowledgments. This work was supported in part by the ECOLEAD project funded by the European Commission. The authors also thank the contributions from their partners.

\section{REFERENCES}

1. Angelov, S., \& Grefen, P. - The $4 \mathrm{~W}$ framework for B2B e-contracting. Int. J. Networking and Virtual Organizations, Vol. 2(No. 1), pp. 78-97. 2003.

2. Afsarmanesh, H.; Camarinha-Matos, L.M. - Future smart organizations: A virtual tourism enterprise, Proceedings of WISE $2000-I^{\text {st }}$ ACM/IEEE International Conference on Web Information Systems Engineering, Vol. 1, pp 456-461, IEEE Computer Society Press, ISBN 0-7695-0577-5, Hong Kong, 19-20 June 2000.

3. Camarinha-Matos, L. M.; Afsarmanesh, H. - Elements of a base VE infrastructure, J. Computers in Industry, Vol. 51, Issue 2, Jun 2003, pp. 139-163.

4. Camarinha-Matos, L. M.; Afsarmanesh, H. - Virtual Enterprise Modeling and Support Infrastructures: Applying Multi-Agent System approaches, in Multi-Agent Systems and Applications, Lecture Notes in Artificial Intelligence LNAI 2086, Springer, ISBN 3-540-42312-5, July 2001.

5. Camarinha-Matos, L. M.; Afsarmanesh, H.; Ollus, M. - Virtual Organizations: Systems and Practices, Springer, Boston, 2005.

6. Camarinha-Matos, L. M.; Cardoso, T. - Selection of partners for a virtual enterprise, in Infrastructures for Virtual Enterprises - Networking Industrial Enterprises, Kluwer Academic Publishers, ISBN 0-7923-8639-6, Oct 1999.

7. Davidrajuh, R.; Deng, Z. Q. - Identifying potential supplier for formation of virtual manufacturing systems, Proceedings of 16th IFIP World Computer Congress 2000, Vol. ITBM, Beijing, China, 21-25 Aug 2000.

8. Kaihara, T. - Supply chain management based on market mechanism in virtual enterprise, in Infrastructures for Virtual Enterprises, Kluwer, Boston, 1999.

9. Li, Y.; Huang, B.Q.; Liu, W. H.; Wu, C.; Gou, H.M. - Multi-agent system for partner selection of virtual enterprises, Proceedings of I6th IFIP World Computer Congress 2000, Vol. ITBM, Beijing, China, 21-25 Aug 2000.

10. McClelland, D. C. - Testing for competence rather than intelligence. American Psychologist, 28, 1-14. 1973.

11. Rabelo, R.; Camarinha-Matos, L.M. - Negotiation in Multi-Agent based dynamic scheduling, Int. Journal on Robotics and CIM, Vol. 11, N. 4, Dec 1994.

12. Rabelo, R.; Camarinha-Matos, L.M.; Vallejos, R. - Agent-based brokerage for virtual enterprise creation in the moulds industry, in E-business and Virtual Enterprises, Kluwer Academic Publishers, ISBN 0-7923-7205-0, pp.281-290, 2000.

13. Rocha, A.; Oliveira, E. - An electronic market architecture for the formation of virtual enterprises, in Infrastructures for Virtual Enterprises, Kluwer, Boston, 1999.

14. Shen W. \& Norrie, D.H. - An agent-based approach for distributed manufacturing and supply chain management, in Globalization of Manufacturing in the Digital Communications Era of the 21st Century:, Jacucci, G. (ed.), Kluwer, Boston, 1998. 